\title{
Source Tracking of Carbapenem-Resistant Enterobacteriaceae Outbreaks in Neonatal Patients Revealed Rivers Contaminated with NDM Genes
}

Jianshjeng Wang ( $\nabla$ wjsxjr@126.com )

Hebei General Hospital

\section{Changfu Yin}

Hebei Medical University

Yuanpeng Lv

Hebei General Hospital

Weiwei Yang

Hebei General Hospital

Peng Zhao

Hebei General Hospital

\section{Research Article}

Keywords: Carbapenem-resistant, Outbreak, Infection prevention, River

Posted Date: April 6th, 2021

DOI: https://doi.org/10.21203/rs.3.rs-395537/v1

License: @ (1) This work is licensed under a Creative Commons Attribution 4.0 International License. Read Full License 


\section{Abstract \\ Background}

Carbapenem-resistant Enterobacteriaceae (CRE) prevalence in neonatal patients continues to rise in china. However, very few epidemiological data are available to uncover the underlying mechanisms for the increase. This study aimed to elucidate their transmission modes and explore potential sources.

\section{Methods}

The genotypic features of CRE isolates were analyzed by PCR and sequencing. The elucidation of transmission and sources involved in electronic medical record review, environmental cultures, screening cultures, and prospective case-control analysis.

\section{Results}

A total of $450 \mathrm{CRE}$ isolates recovered from 369 infant patients were genotypically characterised. Carbapenemase genes NDM-1, KPC-2 and IMP-4 accounted for $86.22 \%(n=388), 8.89 \%(n=40)$ and $4.44 \%(n=20)$, respectively. After implementation of intensified control measures, CRE rectal prevalence rates in both the $0-3$ days and the 4-28 days age group were decreased from $15.53 \%(100 / 644)$ and $27.37 \%(52 / 190)$ to $11.78 \%(51 / 433)$ and $19.59 \%(19 / 97)(P=0.082$ and $P=0.148)$, respectively. Multivariate analysis showed that gastric lavage (odds ratio [OR]) 3.09, 95\% confidence interval [Cl] 1.49-6.39) and enema (OR $2.84,95 \% \mathrm{Cl} 1.65-4.91)$ were independent risk factors for rectal colonisation in the $0-3$ days age group, whereas Cephalosporins (OR 2.12, 95\% Cl 1.14-3.94), gastric lavage (OR 3.44, 95\% Cl 1.44-8.22) and residence in rural area (OR 2.22, 95\% $\mathrm{Cl} 1.19-4.13$ ) were independently associated with rectal colonization in the 4-28 days age group. The strict management of aseptic procedures eventually halted the silent spread. The transmission tracking of neonatal NDM-1 strains outbreaks revealed rivers contaminated with NDM genes.

\section{Conclusions}

Continued vigilance for importations should be maintained to reduce CRE threat. Community awareness raising campaigns are urgently needed to reduce CRE threat.

\section{Introduction}

The ever-increasing carbapenem-resistant Enterobacteriaceae (CRE) has become an extremely intractable problem. Recent data from the CHINET surveillance system in China showed that imipenem-resistance rate of Klebsiella pneumoniae (KP) increased from $3.0 \%$ in 2005 to $25 \%$ in 2018 [1]. The annual upward trend was also obtained from the China Antimicrobial Resistance Surveillance System (http://www.carss.cn/), which further showed that the annual isolation rate of carbapenem-resistant KP (CRKP) in 2014-2019 was 7.2/8.5/9.3/9.1/10.0/10.1 (\%) for pediatric and newborn patients. However, very few detailed epidemiological data are available to uncover the underlying mechanisms for the increase.

In this context, we sought to determine the potential sources and the transmission modes of CRE in neonatal patients by performing a series of integrated analysis of clinical, epidemiologic, microbiologic and molecular data.

\section{Materials And Methods Study design and definition}

The study was conducted in Hebei General Hospita, a 1830-bed tertiary care hospital located in Hebei, China. The study population was stratified by age as follows: 0-3 days old (DO), 4-28 DO, and 29 D0-1 year old (YO) for infant patients. The demographic, clinical, and epidemiological data were collected via electronic medical record review. A CRE case was defined by the isolation of 
CRE in any biological sample obtained from the patient. Infection and colonization were classified as either community acquired or healthcare associated, according to the criteria established by the U.S. Centers for Disease Control and Prevention (CDC) [2]. Patients with known CRE acquisition prior to admission or found to be positive in the first $48 \mathrm{~h}$ of admission were regarded as imported cases.

\section{Microbiological methods}

Various clinically indicated samples, screening samples (stool, rectal/perirectal swabs and amniotic fluid), and environmental samples were taken to detect isolates with reduced susceptibility to carbapenems. Bacterial identification and susceptibility testing were routinely performed using the Vitek 2 automated system (bioMe'rieux) and interpreted following the criteria of the latest Clinical Laboratory Standards Institute (CLSI) document. If necessary, MALDI-TOF MS was used for species verification. Meropenem and imipenem susceptibilities were also performed by the Kirby-Bauer disk diffusion method for culture screening purpose. Carbapenem resistance was defined as nonsusceptibility to imipenem or/and meropenem or carbapenemase genes positive. E. coli ATCC 25922 and P. aeruginosa ATCC 27853 were used as quality controls.

\section{Molecular methods}

Resistance genes were performed by PCR and sequencing, which included genes conferring resistance to class $A, B, C$ and $D \beta$ lactam agents [3-5], and gene encoding resistance to colistin (MCR-1) [6]. Bacterial typing was carried out by multilocus sequence typing (MLST) using primers listed in the online databases ( https://bigsdb.pasteur.fr/klebsiella/klebsiella.html for KP and https://enterobase.warwick.ac.uk/species/ecoli/allele for E.coli). Clone relationships were performed by enterobacterial repetitive intergenic consensus sequence-based PCR (ERIC-PCR) fingerprinting [7]. Plasmid incompatibility group was determined by a PCRbased replicon typing (PBRT) method [8, 9].

\section{Conjugation experiment}

To determine the transferability of carbapenemase genes, a conjugation experiment was carried out in mixed broth cultures at $35^{\circ} \mathrm{C}$ [10]. Carbapenemase positive strains served as donors. Azide-resistant E. coli J53 was used as recipient. Transconjugants were selected on eosin methylene blue agar containing azide $(200 \mu \mathrm{g} / \mathrm{ml})$ and meropenem $(1 \mathrm{mg} / \mathrm{L})$ and inspected by PCRs.

\section{Intervention measures}

No special guidelines regarding CRE prevention in China existed due to the lack of evidence-based epidemiology. Basic infection control measures for CRE employed were based primarily on experience with other multi-drug resistant organisms, which included hand hygiene, contact precautions and/or isolation precautions, healthcare staff education, environmental cleaning and disinfection, aseptic procedures, and antimicrobial stewardship policies.

When recognizing outbreaks, an infection control task force was immediately formed, and multidisciplinary meetings were frequently conducted to identify additional effective control measures based on clinical observations, epidemiological surveys and molecular evidence.

Monthly environmental surveillance cultures, including air sedimentation cultures, hand swab cultures, and environmental swab cultures which involved high-use medical devices, frequently touched common areas, and areas surrounding the patients, were routinely performed in affected wards, especially ICU, maternity, neonatal, and pediatric wards. Amniotic fluid and rectal swabs or feces from pregnant women were also collected during study period. These screenings were most likely based on the following significant assumptions: (i) Prior to 2015, the neonatal ward and ICU were located on the same floor, and both wards experienced the endemicity of KPC-2 KP strains; (ii) Pregnant women with suspected intrauterine infections might carry these bacteria, which transmitted to the newborns during delivery; (iii) Junior medical staff generally rotated on a regular basis between neonatal and pediatric wards, and their movement may be a contributory factor.

To confirm whether infant patients carried CRE in the intestine, we initiated a first prospective rectal screening for the presence of CRE on their admission and during hospitalization during the period from 20th of October 2015 to 30th of April 2017. To observe the efficacy of newly instituted prevention measures on the neonatal colonization, a second prospective rectal surveillance for CRE colonization was performed from September 15, 2017 to April 30, 2018. 


\section{Sampling sites}

The Hutuo River in Hebei Province starts from Houciao Village in Pingshan County and flows eastward into the Bohai Sea. The Gangnan Reservoir and Huangbizhuang Reservoir, located on the main stream of Hutuo River, are the only water sources for industry, agriculture and daily life in this region. The Huangbizhuang Reservoir, 28 km downstream of the Gannan Reservoir, also receives water from Yehe River (Fig. 1). Starting from the Huangbizhuang Reservoir, the Hutuo River runs through 13 counties, 64 towns and 436 villages. These rivers are closely monitored by the local government and do not directly receive untreated human sewage discharge.

Water samples were collected at five sites along Hutuo River (Fig. 1) during late July and early August of 2017. At each sampling site, $5000 \mathrm{ml}$ of water samples were collected in sterile bottles and transported directly to the laboratory for processing.

\section{Statistical analysis}

Data were presented as the mean $\pm S D$, number, percentage, or frequency. Student's t test or Mann-Whitney U test and Chi-squared or Fisher's exact test were used to compare qualitative and categorical variables, respectively. All variables with a $P$ value $\leq 0.1$ in the univariate analysis or that were known risk factors for CRE were included in a backward selection multivariable logistic regression model to identify risk factors independently associated with CRE colonization. Odds ratios (OR) with $95 \%$ confidence intervals $(\mathrm{Cl})$ were calculated. All tests were two-tailed, with a $P$ value of $<0.05$ considered statistically significant. All analyses were performed using SPSS software.

\section{Results}

\section{Genotypic characterization of neonatal and pediatric CRE isolates}

A total of $163 \mathrm{CRE}$ isolates were recovered from clinical samples of 155 infant patients under the age of 1 year admitted in both neonatal and pediatric wards of our hospital during the period from April 2013 to May 2018. CRE prevalence especially occurred in newborns under three days, accounting for $71.78 \%(117)$. KP $(95.71 \%, 156)$ was the most prevalent organism, followed by E.coli $(3.07 \%, 5)$ (Table 1). The most frequently identified carbapenemase gene was NDM-1 $(63.19 \%, 103)$, followed by KPC-2 $(24.54,40)$, and IMP-4 $(12.27 \%, 20)$.

Of the 1,650 patients who were prospectively screened for CRE colonization during the two study periods (948 in the first period and 702 in the second period), 257 patients were identified to be colonized by carbapenem-resistant organisms (CROs) in which 244 cases were carried with NDM-1 producing Enterobacteriaceae strains. Among these colonized patients, 30 (12.3\%) had developed pulmonary infections with these organisms. Co-carriage of two NDM-1 strains was found in $43(17.12 \%)(26$ (15.57\%) of 167 in the first period vs $17(22.08 \%)$ of 77 in the second period, $P=0.215)$ of the 244 CRE patients. CRE rectal prevalence rate in the 29 DO-1 YO groups were significantly decreased from $14.29 \%(15 / 105)$ in the first period to $4.07 \%(7 / 172)$ in the second period $(P=0.002)$. The rectal prevalence rates of NDM-1 strains in both the 0-3 DO and the 4-28 DO groups were decreased, but no statistical significances were reached $(15.53 \%, 100 / 644$ vs $11.78 \%, 51 / 433, P=0.082$ and $27.37 \%, 52 / 190$ vs $19.59 \%, 19 / 97, P=$ 0.148 , respectively).

In the first period, 193 CRE were isolated, including 182 NDM-1 CPKP of 7 STs, with ST20 $(40.11 \%, 73)$, ST2068 $(36.26 \%, 66)$, and ST36 $(19.23 \%, 35)$ being the 1st, 2nd and 3rd most common types, 8 NDM-1 E.coli of 6 STs and 3 NDM-1 Enterobacter cloacae strains. Although transmission events continued to occur during the second period, isolates of ST types or species were completely different from those in the first period, and all isolates were NDM-1-producers consisting of 65 CPKP of 8 STs with a predominance of ST17 $(73.85 \%, 48)$ and 29 E.coli of 4 STs with ST325 $(89.62 \%, 26)$ as the major type (Table 1$)$.

\section{Neonatal outbreak evolution and infection control measures}

In April 2013, a sporadic epidemic of NDM-1 ST39 KP occurred in neonatal ward, involving 13 cases less than 3 days old. The strain was initially isolated in March 2013 from a 5-month-old child with community-acquired pneumonia in the pediatric ward and 
might subsequently be transmitted to the neonatal ward via rotating medical staff. In July 2014, KPC-2 ST11 KP and IMP-4 ST307 KP clones were introduced into the ward and then spread rapidly, affecting 40 and 17 neonatal cases, respectively. Despite the implementation of basic infection control practices, especially enhanced environmental disinfection and reinforced hand hygiene before and after patient contact, new cases were still emerging. Routine environmental screening demonstrated a heavy environmental contamination with CROs (Supplementary Table 1 and Supplementary Figure 1). ERIC analysis of patients' isolates and environmental isolates recovered from both the neonatal ward and adult ICU showed that some Aci.baumannii strains and ST11 KP clones were, respectively, closely related (Supplementary Figure 1), suggesting an important route of 'non-patient transfer' transmission between wards. So a new ward was opened in another building at the end of 2014 to receive new admissions. Those who had been affected remained in the original ward until they were discharged. Extensive environmental screening cultures were performed one month before and after the opening of the new ward, but did not yield any CROs.

In November 2015, the new unit was partially closed to external admissions due to NDM-1 ST20 and ST2068 KP. To determine the probable sources of the outbreak strains, 342 stool or perianal samples and 286 amniotic fluid samples collected from 342 pregnant women with suspected intrauterine infection in the maternity ward were screened for CROs, but no isolates were identified to be carbapenem-resistant. Therefore, the likely route of CRO through vertical transmission from the mothers at birth was excluded. The fact that there were no CROs circulating in the maternity ward and that no CROs had been isolated from routine obstetric environmental screenings did not support the transmission from maternity to neonatal ward. Next, we speculated that asymptomatic carriers might bring these organisms into the unit, so active rectal screening cultures were initiated for infant patients under one year old.

In October 2016, a new NDM-1 ST36 KP clone emerged and spread, affecting 33 cases. In March 2017, the initial results of rectal cultures were fed back to the ward, emphasizing that the frequent community importations of asymptomatically colonised patients and subsequent transmissions should be considered as key elements for intervention. A series of intensified measures were then instituted and implemented, including enhanced environmental disinfection with chlorine-based compound in frequency and extent, especially the carriers' surroundings, the reduction of the total beds of the room from 14 to 10 , increase of staff numbers, and reinforcement of hand hygiene compliance. Afterward, the number of clinical CRE isolates decreased drastically, and the CROs ceased to be detected from the ward environment.

In September and October 2017, two new NDM-1 strains of ST325 E.coli and ST17 KP were separately introduced and silently spread, leading to colonization in 23 and 40 cases, respectively. This suggested that these measures were far from sufficient to avoid silent transmissions. We then separately performed prospective matched case-control studies to determine neonatal risk factors associated with rectal colonization of NDM-1-producing Enterobacteriaceae in the 0-3 DO group and the 4-28 DO group. In the multivariate analysis, we found that gastric lavage and enema were independent risk factors for colonization in 0-3 DO group, whereas gastric lavage was a risk factor independently associated with colonization in 4-28 DO group (Table 2). These results strongly suggested that improperly sterilized procedures had probably direct causal role in neonatal colonization and transmission. So strict aseptic procedures were emphasized and monitored to insure stringent compliance in caring for high-risk patients. With the addition of these interventions, sporadic new CRE cases were still identified in the following years, but no outbreaks occurred.

\section{Neonatal outbreak tracking revealed rivers contaminated with NDM genes}

A detailed analysis of patient admission data combined with cultures and molecular typing revealed that multiple or repeated independent introductions of diverse NDM-1 producers into the neonatal ward direct from the community through colonized/infected infant patients, especially patients older than 3 days, resulted in continued clone transmission events (Figure 2). None of these colonized cases and their family members had any prior history of foreign travel to, or previous exposure to, known areas of high NDM endemicity. Multivariate analysis showed that residence in rural area was an independent risk factor, so we tried to put patients' home addresses on a regional map to determine the scope of CRE endemicity in the community. We found that most patients lived in the vicinity of Hutuo River or tributaries where people frequently visited in summer (Supplementary Figure 2). Significantly increased incidences of carriage with community-acquired NDM-1 strains were observed during summers 
(river contact seasons) compared to winters (no river contact seasons) $(16(24.62 \%)$ of 65 vs $15(12 \%)$ of $125, P=0.026)$. The obvious seasonality in NDM carriage rates might be, in large part, associated with increased chances of exposure to the rivers.

To determinate the rivers as the likely sources, extensive water samplings were carried out. A total of $83 \mathrm{CRO}$ s were recovered and screened for the presence of common carbapenemase genes (Supplementary Table 2 and Table 3). NDM producers could only be isolated at sampling sites C, D, and E (Figure 1). NDM-1 isolates (Aci.baumannii, Aci.Iwoffiii, KP of ST37 and ST1306), NDM-5 E.coli of ST167, IMI-3 E.cloacae, OXA-24 Aci.Iwoffiii and blaOXA-24, and blaOXA-51 co-producing Aci.baumannii isolates were detected in the rivers. The antimicrobial susceptibility profiles of water NDM isolates were similar to those of clinical isolates, showing high-level resistance to penicillins and cephalosporins but susceptibility to aminoglycosides and quinolones. ERIC analysis showed that water isolates of Aci.Iwoffiii were unrelated to each other, suggesting horizontal plasmid transmission (Figure 3A), while ST37 KP and ST167 E.coli isolates recovered from river were genetically related to those isolated from patients (Figure 3B and 3C). Water ST37 isolate harbored extended-spectrum beta-lactamase genes (ESBLs) (SHV-31/CTX-15) and IncX3 replicon, whereas neonatal ST37 isolate possessed TEM-1/SHV-11/CTX-M-14 and A/C replicon. Both water and clinical isolates of ST167 E.coli had the same ESBLs (TEM-1/CTX-15) and IncFII replicon, but colistin resistance gene MCR-1 was detected only in the latter, suggesting that this strain likely originated from the river and had underwent a complex evolutionary process before transmission to humans.

\section{Transferability of water isolates via conjugation}

Water isolates of ST1306 NDM-1 KP and ST167 NDM-5 E.coli, and NDM-1Aci.Iwoffii isolates were able to transfer, while the ST37 NDM-1 KP strain and one Aci.Iwoffii isolate that co-produced both NDM-1 and OXA-24 failed to transfer.

\section{Discussion}

In the study, we found that multiple independent introductions of diverse NDM-1 strains from the community and the resultant equipment or environmental contamination potentially contributed to their spread in the neonatal ward. Although basic control measures had been strictly implemented throughout the study period, they were fundamentally inadequate to curb their transmission. Intensified measures developed on the basis of a comprehensive understanding of their transmission had proven to be effective. Neonatal outbreak investigation and transmission tracking revealed that the rivers might serve as large NDM pools for the transmission in the community.

The neonatal ward had experienced several outbreaks of NDM-1 strains and finally been terminated through multifaceted intervention measures based on more accurate knowledge of the transmission events. The complete success was attributed to the following three key factors: (i) Active rectal surveillance cultures combined with efficient bacterial typing methods could give more complete transmission information that assisted in allowing for timely targeted control measures to be taken. Previously, CRE were detected only based on clinical samples while the transmission caused by colonization was almost completely ignored, leading to environmental contamination. (ii) Another important component in the success of the intervention was to reduce the workload and improve compliance with control measures. (iii) Risk assessment could reveal equipment- or device-associated transmission and enable precise interventions [11-14], even sometimes without any bacterial evidence $[13,14]$. Our findings indicated that accurate evaluation of the risk factors that had contributed to neonatal acquisitions could identify potential factors for silent transmission and further improve infection control measures.

Walsh et al. reported that NDM organisms largely occurred and disseminated in the community primarily through waterborne and foodborne transmission [10]. The diffusion of NDM-1 or -5 in China was mainly mediated by X3 plasmid [15-20] and their sources in the community were identified as animals [15, 16], migratory birds [17], fresh vegetables [18], retail meat [19], multiple commercial farms [16, 20], and rivers [21, 22]. Some NDM strains also carried MCR-1 [19, 20, 24], which originated in animals and had spread to humans [6]. This study revealed vast, unrecognized NDM contaminated rivers accessible to people. KPC-2 bacteria and OXA-23 Aci.baumannii occurred almost exclusively in healthcare settings, while NDM-1 Aci.Iwoffii and OXA-24 Aci.baumannii appeared predominantly in river environment. How these strains occurred and evolved in the rivers remained to be further explored. 
Some water NDM isolates were able to transfer their NDM plasmids via conjugation, and they could serve as potential vectors or reservoirs for transferring themselves and/or NDM genes. Our findings strongly supported the hypothesis that NDM strains in the rivers could be transferred to families where babies could potentially obtain through daily life contact with their family members who acquired after frequent exposure to or contact with NDM contaminated rivers, or through the use of contaminated washing materials (clothes, bed linen, etc.) washed in the same rivers. Once colonized and adapted to the new human environment, these NDM organisms could further propagate or/and transfer their mobile resistance genes to the microbes that inhabited the human body, which were then introduced into healthcare settings or transmitted to their close contacts in the community.

\section{Conclusions}

CRE would become a persistent problem in the future, largely due to the continuing risk of importation pressures from the community. Hence, continued vigilance and comprehensive epidemiology studies specific for institutions or regions are urgently needed to address the future CRE challenge.

\section{Abbreviations}

CRE: Carbapenem-resistant Enterobacteriaceae; CRO: Carbapenem-resistant organism; KP: Klebsiella pneumoniae; CRKP: Carbapenem-resistant Klebsiella pneumoniae; DO: Days old; YO: Year old

\section{Declarations}

\section{Acknowledgments}

We are indebted to clinical staff of the hospital for their extensive support in the investigation of outbreaks; and to Guo yumei at Shijiazhuang Center for Disease Control and Prevention for technical assistance with ERIC-PCR analysis.

\section{Authors' contributions}

Jiansheng Wang and Changfu Yin had made substantial contributions to the conception and design of the study, the acquisition, analysis and interpretation of data. Jiansheng Wang had been responsible for critically drafting and revising the manuscript for all critical intellectual content. Yuanpeng Lv, Weiwei Yang and Peng Zhao have made substantial contributions to sample collections, experiments, and data acquisition and interpretation. All authors read and approved the final manuscript.

\section{Funding}

This work was funded by Hebei medical applicable technology tracking project (grant number GL2012049).

\section{Availability of data and materials}

The datasets used and analysed during the current study are available from the corresponding author on reasonable request.

\section{Ethics approval and consent to participate}

Protocols for collection of samples as well as the experiment plan and all methods were performed in accordance with the guidelines and regulations of Hebei general hospital and approved by the institutional ethical committees. Informed consent was obtained from participants or parents prior to their involvement. 


\section{Consent for publication}

All authors read and approved the final manuscript and gave consent for publication.

\section{Competing interests}

The authors have no competing interests.

\section{References}

1. Hu F, Guo Y, Yang Y, Zheng Y, Wu S, Jiang X, Zhu D, Wang F; China Antimicrobial Surveillance Network (CHINET) Study Group. Resistance reported from China antimicrobial surveillance network (CHINET) in 2018. Eur J Clin Microbiol Infect Dis. 2019;38(12):2275-2281.

2. Horan TC, Andrus M, Dudeck MA. CDC/NHSN surveillance definition of health care-associated infection and criteria for specific types of infections in the acute care setting. Am J Infect Control. 2008;36(5):309-32.

3. Queenan AM, Bush K. Carbapenemases: the Versatile-Lactamases. Clin Microbiol Rev. 2007;20(3):440-58.

4. Dallenne C, Da Costa A, Decré D, Favier C, Arlet G. Development of a set of multiplex PCR assays for the detection of genes encoding important \-lactamases in Enterobacteriaceae. J Antimicrob Chemother. 2010;65(3):490-5.

5. Sidjabat H, Nimmo GR, Walsh TR, Binotto E, Htin A, Hayashi Y, Li J, Nation RL, George N, Paterson DL. Carbapenem resistance in Klebsiella pneumoniae due to the New Delhi Metallo- $\beta$-lactamase. Clin Infect Dis. 2011;52(4):481-4.

6. Liu YY, Wang Y, Walsh TR, Yi LX, Zhang R, Spencer J, Doi Y, Tian G, Dong B, Huang X, et al. Emergence of plasmid-mediated colistin resistance mechanism MCR-1 in animals and human beings in China: a microbiological and molecular biological study. Lancet Infect Dis. 2016;16(2):161-8.

7. Cartelle M, del Mar Tomas M, Pertega S, Beceiro A, Dominguez MA, Velasco D, Molina F, Villanueva R, Bou G. Risk factors for colonization and infection in a hospital outbreak caused by a strain of Klebsiella pneumoniae with reduced susceptibility to expanded-spectrum cephalosporins. J Clin Microbiol. 2004;42(9):4242-9.

8. Carattoli A, Bertini A, Villa L, Falbo V, Hopkins KL, Threlfall EJ. Identification of plasmids by PCR-based replicon typing. J Microbiol Methods. 2005;63(3):219-28.

9. Compain F, Poisson A, Le Hello S, Branger C, Weill FX, Arlet G, Decré D. Targeting relaxase genes for classifification of the predominant plasmids in Enterobacteriaceae. Int J Med Microbiol. 2014;304(3-4):236-42.

10. Walsh TR,Weeks J, Livermore DM, Toleman MA.. Dissemination of NDM-1 positive bacteria in the New Delhi environment and its implications for human health: an environmental point prevalence study. Lancet Infect Dis. 2011;11(5):355-62.

11. Kim S, Russell D, Mohamadnejad M, Makker J, Sedarat A, Watson RR, Yang S, Hemarajata P, Humphries R, Rubin Z, et al. Risk Factors Associated with the Transmission of Carbapenem-Resistant Enterobacteriaceae via Contaminated Duodenoscopes. Gastrointest Endosc. 2016;83(6):1121-9.

12. Epstein L, Hunter JC, Arwady MA, Tsai V, Stein L, Gribogiannis M, Frias M, Guh AY, Laufer AS, Black S, et al. New Delhi metalloB-lactamase-producing carbapenem-resistant Escherichia coli associated with exposure to duodenoscopes. JAMA. 2014;312(14):1447-55.

13. Potron A, Bernabeu S, Cuzon G, Pontiès V, Blanchard H, Seringe E, Naas T, Nordmann P, Dortet L. Analysis of OXA-204 carbapenemase-producing Enterobacteriaceae reveals possible endoscopy-associated transmission, France, 2012 to 2014. Euro Surveill. 2017;22(49):17-48.

14. Humphries RM, Yang S, Kim S, Muthusamy VR, Russell D, Trout AM, Zaroda T, Cheng QJ, Aldrovandi G, Uslan DZ, et al. Duodenoscope-Related Outbreak of a Carbapenem-Resistant Klebsiella pneumoniae Identified Using Advanced Molecular Diagnostics. Clin Infect Dis. 2017;65(7):1159-1166. 
15. Li J, Bi Z, Ma S, Chen B, Cai C, He J, Schwarz S, Sun C, Zhou Y, Yin J, et al. Inter-host Transmission of CarbapenemaseProducing Escherichia coli among Humans and Backyard Animals. Environ Health Perspect. 2019;127(10):107009.

16. Wang Y, Zhang R, Li J, Wu Z, Yin W, Schwarz S, Tyrrell JM, Zheng Y, Wang S, Shen Z, et al. Comprehensive resistome analysis reveals the prevalence of NDM and MCR-1 in Chinese poultry production. Nat Microbiol. 2017;2:16260.

17. Liao X, Yang RS, Xia J, Chen L, Zhang R, Fang LX, Lei F, Song G, Jia L, Han L, et al. High colonization rate of a novel carbapenem-resistant Klebsiella lineage among migratory birds at Qinghai Lake, China. J Antimicrob Chemother. 2019;74(10):2895-2903.

18. Liu BT, Zhang XY, Wan SW, Hao JJ, Jiang RD, Song FJ. Characteristics of Carbapenem-Resistant Enterobacteriaceae in Readyto-Eat Vegetables in China. Front Microbiol. 2018;9:1147.

19. Zhang Q, Lv L, Huang X, Huang Y, Zhuang Z, Lu J, Liu E, Wan M, Xun H, Zhang Z, et al. Rapid Increase in CarbapenemaseProducing Enterobacteriaceae in Retail Meat Driven by the Spread of the bla NDM-5-Carrying IncX3 Plasmid in China from 2016 to 2018. Antimicrob Agents Chemother. 2019;63(8):e00573-19.

20. Xiang R, Zhang AY, Ye XL, Kang ZZ, Lei CW, Wang HN. Various sequence types of Enterobacteriaceae carrying blaNDM-5 gene from commercial chicken farms in China. Antimicrob Agents Chemother. 2018;62(10):e00779-18.

21. Zhao Q, Berglund B, Zou H, Zhou Z, Xia H, Zhao L, Nilsson LE, Li X. Dissemination of bla(NDM-5) via IncX3 plasmids in carbapenem-resistant Enterobacteriaceae among humans and in the environment in an intensive vegetable cultivation area in eastern China. Environ Pollut. 2021;273:116370.

22. Khan H, Miao X, Liu M, Ahmad S, Bai X. Behavior of last resort antibiotic resistance genes (mcr-1 and bla(NDM-1)) in a drinking water supply system and their possible acquisition by the mouse gut flora. Environ Pollut. 2020;259:113818.

\section{Tables}

Table 1 Distribution of carbapenem-resistant Enterobacteriaceae (CRE) isolates in infant patients

\begin{tabular}{|c|c|c|c|c|c|c|c|c|c|c|c|c|c|c|}
\hline & \multicolumn{5}{|c|}{ 0-3 days age group } & \multicolumn{5}{|c|}{ 4-28 days age group } & \multicolumn{4}{|c|}{ 29-365 days age group } \\
\hline & $\begin{array}{l}\text { Sequence } \\
\text { types of KP }\end{array}$ & No. & $\begin{array}{l}\text { Sequence } \\
\text { types of EC }\end{array}$ & No. & $\begin{array}{l}\text { Other } \\
\text { CRE }\end{array}$ & $\begin{array}{l}\text { Sequence } \\
\text { types of KP }\end{array}$ & No. & $\begin{array}{c}\text { Sequence } \\
\text { types of EC }\end{array}$ & No. & $\begin{array}{l}\text { Other } \\
\text { CRE }\end{array}$ & $\begin{array}{c}\text { Sequence } \\
\text { types } \\
\text { of KP }\end{array}$ & No. & $\begin{array}{c}\text { Sequence } \\
\text { types } \\
\text { of EC }\end{array}$ & No. \\
\hline $\begin{array}{l}\text { First stool } \\
\text { screening }\end{array}$ & $\begin{array}{l}\text { ST13 } \\
\text { ST20 } \\
\text { ST36 } \\
\text { ST2068 }\end{array}$ & $\begin{array}{l}2 \\
49 \\
19 \\
41\end{array}$ & ST1 & 1 & $2^{a}$ & $\begin{array}{l}\text { ST13 } \\
\text { ST20 } \\
\text { ST36 } \\
\text { ST2068 } \\
\text { ST37 } \\
\text { ST45 }\end{array}$ & $\begin{array}{l}3 \\
16 \\
14 \\
20 \\
1 \\
1\end{array}$ & $\begin{array}{l}\text { ST767 } \\
\text { ST167 } \\
\text { ST524 }\end{array}$ & $\begin{array}{l}1 \\
1 \\
1\end{array}$ & $1^{\mathrm{a}}$ & $\begin{array}{l}\text { ST20 } \\
\text { ST36 } \\
\text { ST2068 } \\
\text { ST2267 }\end{array}$ & $\begin{array}{l}8 \\
2 \\
5 \\
1\end{array}$ & $\begin{array}{l}\text { ST196 } \\
\text { ST167 } \\
\text { ST4542 }\end{array}$ & $\begin{array}{l}1 \\
2 \\
1\end{array}$ \\
\hline $\begin{array}{l}\text { Second stool } \\
\text { screening }\end{array}$ & $\begin{array}{l}\text { ST17 } \\
\text { ST23 } \\
\text { ST20 }\end{array}$ & $\begin{array}{l}33 \\
4 \\
3\end{array}$ & ST325 & 20 & & $\begin{array}{l}\text { ST17 } \\
\text { ST4 } \\
\text { ST2068 }\end{array}$ & $\begin{array}{l}14 \\
4 \\
1\end{array}$ & ST325 & 6 & & $\begin{array}{l}\text { ST17 } \\
\text { ST23 } \\
\text { ST45 } \\
\text { ST685 } \\
\text { ST36 }\end{array}$ & $\begin{array}{l}1 \\
1 \\
1 \\
1 \\
2\end{array}$ & $\begin{array}{l}\text { ST398 } \\
\text { ST469 } \\
\text { ST83 }\end{array}$ & $\begin{array}{l}1 \\
1 \\
1\end{array}$ \\
\hline $\begin{array}{c}\text { Clinical } \\
\text { specimens* }\end{array}$ & $\begin{array}{l}\text { ST11 } \\
\text { ST13 } \\
\text { ST20 } \\
\text { ST36 } \\
\text { ST39 } \\
\text { ST307 } \\
\text { ST2068 } \\
\text { ST17 } \\
\text { ST23 } \\
\text { ST1786 }\end{array}$ & $\begin{array}{l}29 \\
1 \\
33 \\
13 \\
13 \\
15 \\
4 \\
2 \\
2 \\
1\end{array}$ & $\begin{array}{l}\text { ST471 } \\
\text { ST469 } \\
\text { ST325 }\end{array}$ & $\begin{array}{l}1 \\
1 \\
1\end{array}$ & $1^{b}$ & $\begin{array}{l}\text { ST11 } \\
\text { ST20 } \\
\text { ST36 } \\
\text { ST2068 } \\
\text { ST17 } \\
\text { ST1655 } \\
\text { ST307 }\end{array}$ & $\begin{array}{l}8 \\
10 \\
2 \\
2 \\
3 \\
1 \\
3\end{array}$ & ST325 & 1 & $1^{\mathrm{a}}$ & $\begin{array}{l}\text { ST11 } \\
\text { ST20 } \\
\text { ST39 } \\
\text { ST36 } \\
\text { ST719 } \\
\text { ST23 } \\
\text { ST307 }\end{array}$ & $\begin{array}{l}3 \\
4 \\
1 \\
3 \\
1 \\
1 \\
1\end{array}$ & ST325 & 1 \\
\hline
\end{tabular}


*: Clinical specimens including 147 bronchial aspirates or aspirating sputa, 14 blood samples and 2 purulent fluids were detected positive for carbapenemase-producing Enterobacteriaceae. KP, Klebsiella pneumoniae; EC, Escherichia coli; ECL, Enterobacter cloacae. a: NDM-1 ECL; b: IMP-4 Klebsiella oxytoca. ST11, KPC-2-producing KP; ST307, IPM-4-producing KP; Other STs were NDM-1 producers except for 2 KPC-2 and NDM-1 co-producing KP of ST20 strains.

Table 2 Risk factors associated with rectal colonization of NDM-1-producing Enterobacteriaceae in neonatal patients

Table 3 Distribution of common carbapenemase genes in river isolates

Organisms $\quad$ Sources $\quad$ OXA-24 OXA-24 and OXA-51 NDM IMI-3

\begin{tabular}{|c|c|c|c|c|}
\hline Acinetobacter baumannii & Gangnan Reservoir & 1 & & \\
\hline & Gangnan Reservoir downstream & 1 & 1 & \\
\hline & Yehe River & & & 1 \\
\hline & Huangbizhuang Reservoir & 1 & 3 & 1 \\
\hline & Chahe River & 2 & & \\
\hline & Yehe River & & & 5 \\
\hline Acinetobacter lwoffi & Huangbizhuang Reservoir & 1 & & 1 \\
\hline & Chahe River & 1 & & \\
\hline
\end{tabular}

\begin{tabular}{lcc} 
Klebsiella pneumoniae & Yehe River & 1 \\
\cline { 2 - 3 } & Chahe River & 1
\end{tabular}




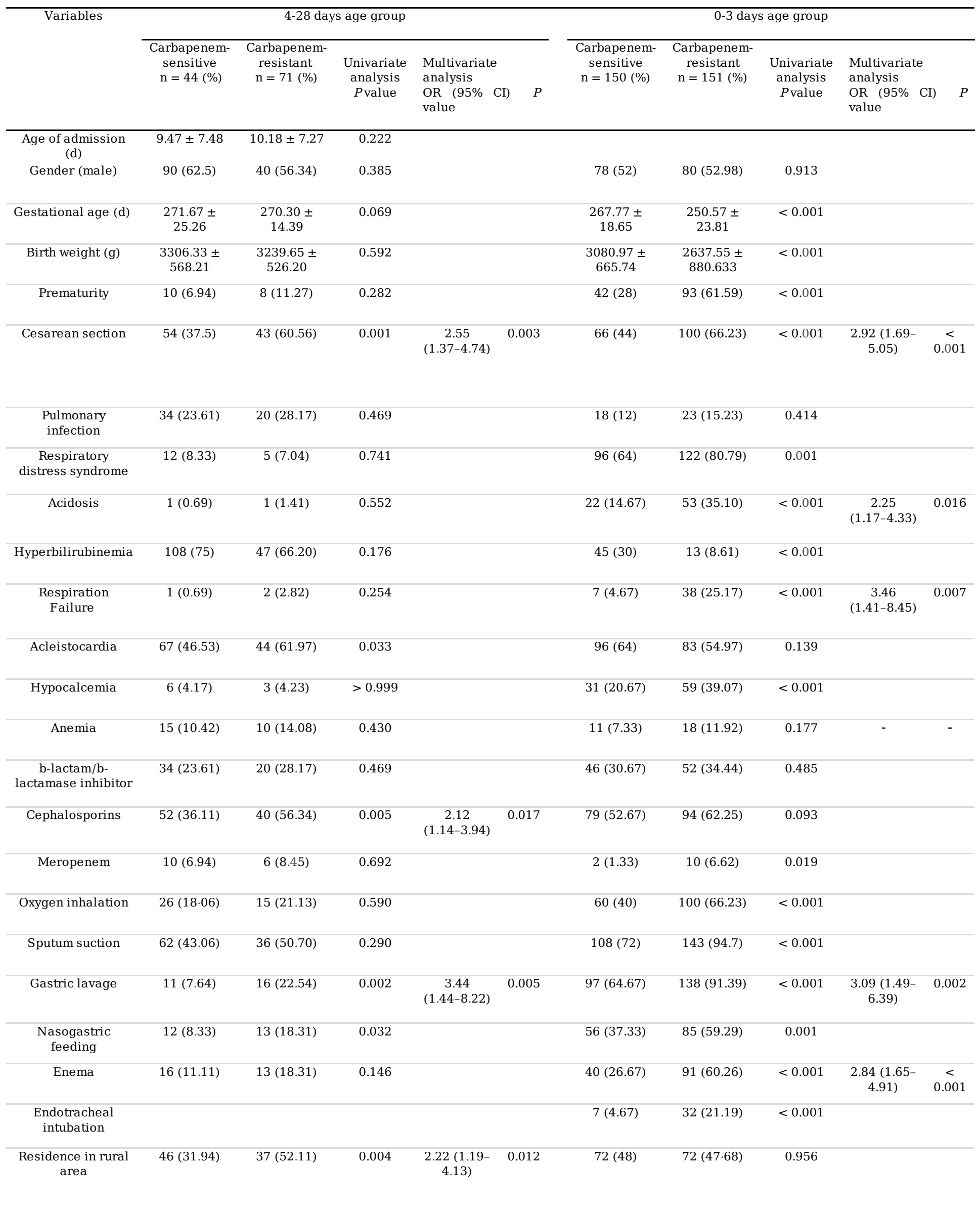




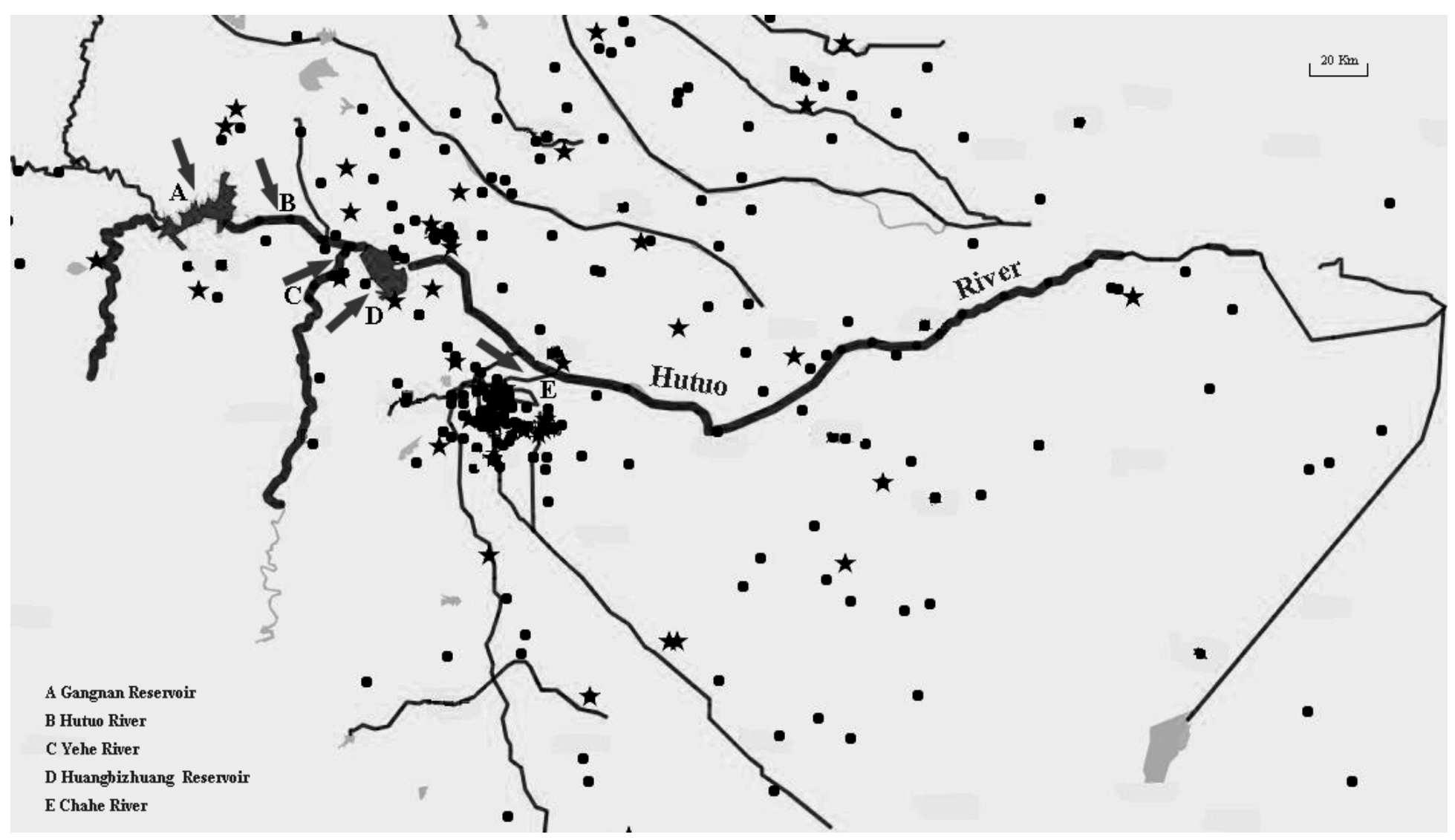

Figure 1

Location of sampling sites and home address-based distribution of infant patients with carbapenemase-producing Enterobacteriaceae (CPE). To explore the scope of CPE transmission in the community, neonatal patients' home addresses were indicated by solid circles and pentacles on the map, which represented hospital-acquired and conmmunity-acquired, respectively. Arrows indicated sample collection points. Note: The designations employed and the presentation of the material on this map do not imply the expression of any opinion whatsoever on the part of Research Square concerning the legal status of any country, territory, city or area or of its authorities, or concerning the delimitation of its frontiers or boundaries. This map has been provided by the authors. 

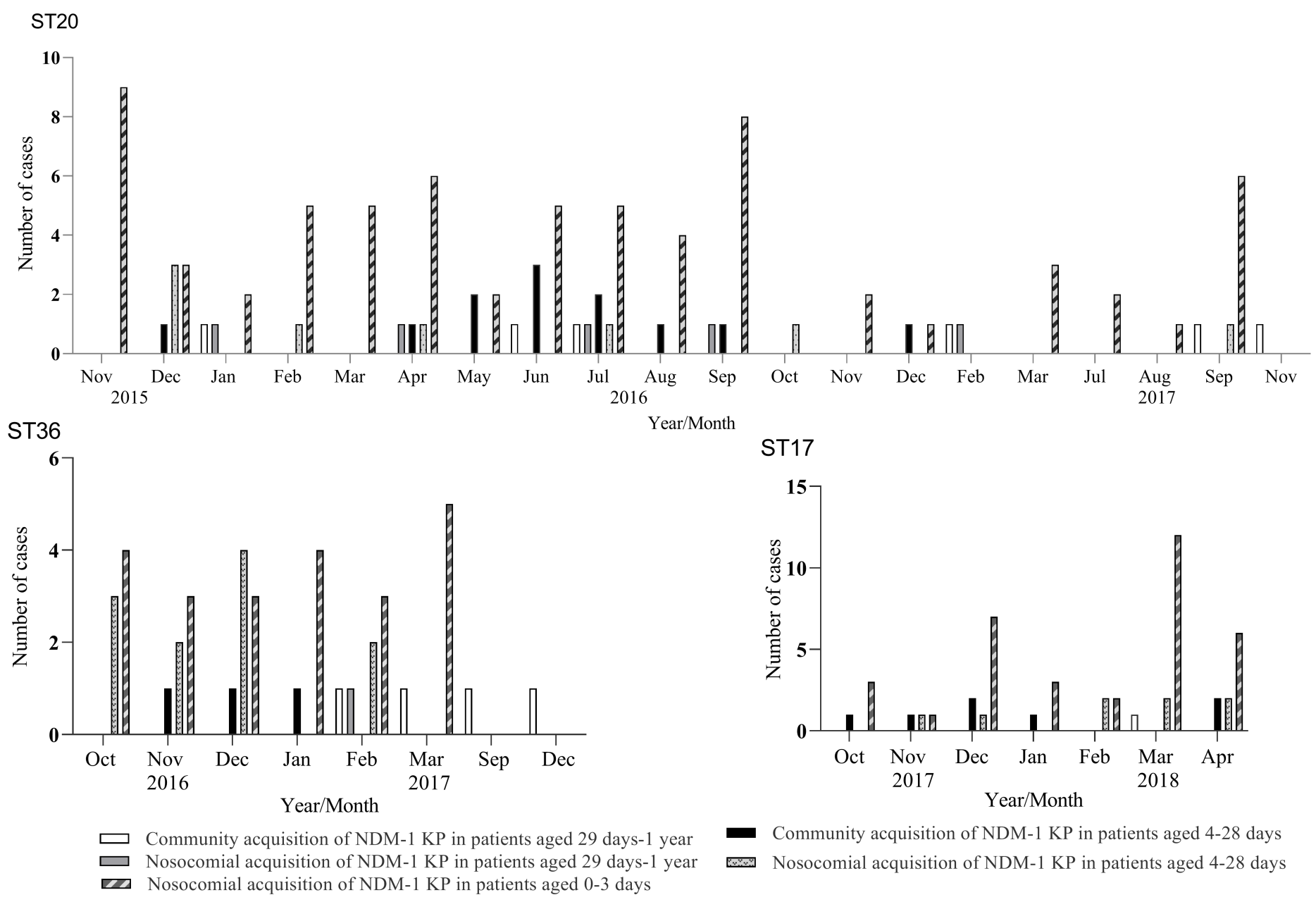

- Community acquisition of NDM-1 KP in patients aged 4-28 days

Nosocomial acquisition of NDM-1 KP in patients aged 4-28 days

Figure 2

Distribution of infant patients with NDM-1 Klebsiella pneumoniae strains of different STs according to months
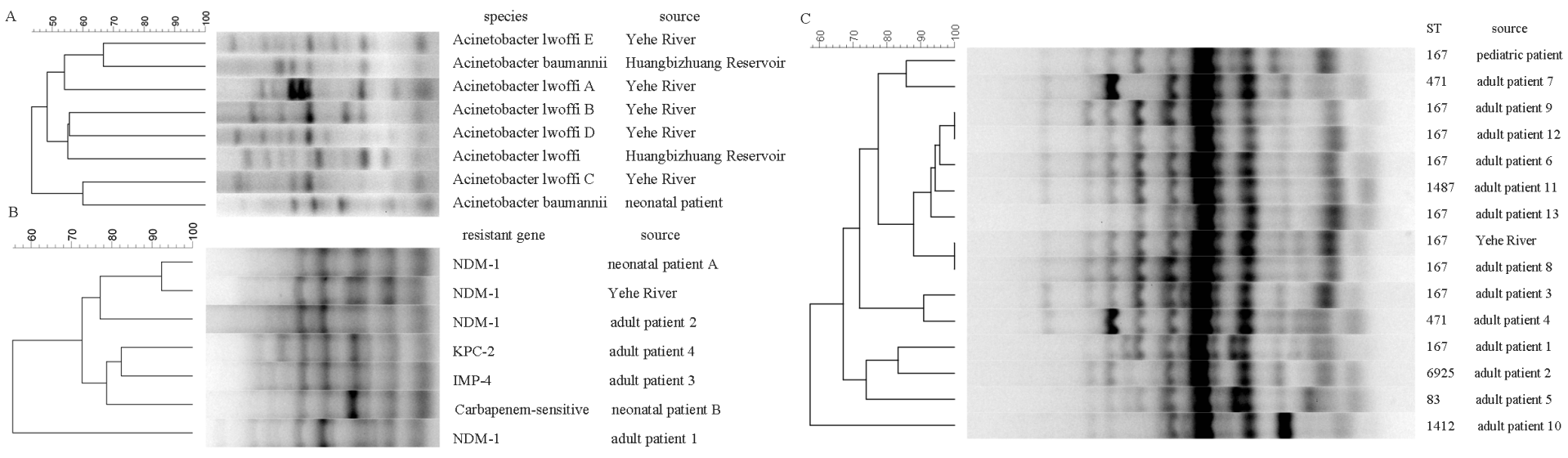

\section{Figure 3}

Genotypic relationships of carbapenem-resistant organisms from rivers and patients. To observe the genetic relationships between clinical and water isolates, adult CPE isolates were also used for genetic analysis. Dendrogram depicting the genetic relatedness among the isolates was obtained by cluster analysis of ERIC profiles according to Dice's similarity coefficient and the unweighted pair-group method with arithmetic mean UPGMA (the position tolerance and optimization were set at 1.0 and $1.5 \%$, respectively). Panel A shows NDM-1 producing Acinetobacter spp.. The fecal isolate from a neonatal patient displayed a banding profile distinct 
from those river isolates. Panel B shows K.pneumoniae isolates of ST37. The river and neonatal patient isolates showed similar banding patterns. Panel C shows NDM-5 producing E.coli isolates. The river and adult patient isolates of ST167 exhibited identical banding patterns.

\section{Supplementary Files}

This is a list of supplementary files associated with this preprint. Click to download.

- Supplementarymaterial.docx 